TITLE:

\title{
Electronic structures of newly designed two-dimensional high- spin organic polymers
}

$\operatorname{AUTHOR}(\mathrm{S})$ :

Ito, Akihiro; Ino, Haruhiro; Tanaka, Kazuyoshi

CITATION:

Ito, Akihiro ...[et al]. Electronic structures of newly designed two-

dimensional high-spin organic polymers. Polyhedron 2009, 28(9-10):

2080-2086

ISSUE DATE:

2009-06-22

URL:

http://hdl.handle.net/2433/123382

\section{RIGHT:}

Copyright (c) 2009 Elsevier; This is not the published version. Please cite only the published version.; この論文は出版社版でありません。引用の 際には出版社版をご確認ご利用ください。 


\title{
Electronic Structures of Newly Designed 2-Dimensional High-Spin Organic Polymers
}

\author{
Akihiro Ito, ${ }^{*}$ Haruhiro Ino, and Kazuyoshi Tanaka \\ Department of Molecular Engineering, Graduate School of Engineering, Kyoto \\ University, Nishikyo-ku, Kyoto, 615-8510, Japan
}

\begin{abstract}
Practical 2-dimensional high-spin organic polymers are newly designed and the electronic structures are examined on the basis of the ab initio 2-dimensional unrestricted Hartree-Fock crystal orbital (UHF-CO) method. The present polymers can be oxidized up to six electrons per unit cell, and it is predicted that the threeelectron and six-electron oxidations per unit cell lead to the high-spin organic polymers having superdegenerate band structures originating from the characteristic non-bonding crystal orbital patterns.
\end{abstract}

Keywords: High-spin organic polymer; Arylamine; Poly(radical cation); Crystal orbital

* Corresponding author. Fax: +81 753832556.

E-mail addresses: aito@scl.kyoto-u.ac.jp (A. Ito) 


\section{Introduction}

Polymer-based organic ferromagnets have been attracting much attention since Mataga's proposal [1]. This is firstly because the design of such polymers is conceptually straightforward and secondly because magnetic interaction through covalent intramolecular pathway can be expected to be strong compared with the known crystalline ferromagnets composed of stable radical molecules [2].

In the polymer-based approach, the entire polymer can be constructed from two units: (i) a spin-containing (SC) unit and (ii) a ferromagnetic coupling (FC) unit [3]. The concept of FC unit is equivalent to appropriate choice of spacer connecting between SC units. To date, 1,3-benzenediyl and/or 1,3,5-benzenetriyl are well-known FC units. When two SC units are connected by 1,3-benzenediyl, the resulting diradical has inevitably two nearly degenerate non-bonding molecular orbitals (NBMOs) (for example, $m$-benzoquinodimethane). These NBMOs distribute over common atoms and therefore strong exchange interactions are expected to occur between two radical spins, leading to a high-spin preference [4]. This mechanism is closely related to the Hund's rule in atomic physics. Employing several kinds of SC units, a lot of polymers and their oligomer model molecules have been investigated along this line $[5,6]$. However, authentic preparation of organic polymer-based ferromagnets still remains questionable [7].

On the other hand, over the past 40 years, a considerable number of theoretical studies have been made on several organic high-spin model polymers. On the analogy of the NBMOs in high-spin molecules, the concept of "superdegeneracy" has been introduced into the half-filled bands for possible ferromagnetic polymers $[8,9]$. Furthermore the elaborate density functional studies have been performed to give more 
reliable information on the electronic structures of ferromagnetic 1-dimensional (1-D) polymers and dendrimers [10].

As is well-known as Mermin and Wagner's theorem, in 1-D spin systems [11], the magnetic long range order cannot be accomplished at finite temperature. Moreover, for practical application of the 1-D magnetic polymer, generation of the spinless sites due to incomplete oxidation or decomposition leads to disappearance of high-spin correlation. Hence, 2-D or 3-D spin network is indispensable to the realization of defect-tolerant high-spin polymers [2].

Recently, we have investigated the polycationic states of a series of oligoarylamines in order to prepare 2-dimensional (2-D) high-spin polymers [12-17]. These stable polycationic species are found to be in their high-spin ground states, probably due to the combination of the strong FC unit (1,3,5-benzenetriyl and 1,3-benzenediyl) and the robust SC unit (semiquinone radical cation of para-phenylenediamine). For example, the trication of the dendritic oligoarylamine $\mathbf{3}$ has quartet ground state [13], and the dication of the macrocyclic oligoarylamine 4 has triplet ground state [14]. When we design the arylamine-based 2-D high-spin polymers, the Mataga-type polymer is inappropriate for the candidate. This is because such a molecular structure does not allow the planar 2-D structure due to the sterically overcrowded molecular structure. On the other hand, polymers $\mathbf{1}$ and $\mathbf{2}$ having meta- and para-phenylene linkage make it possible to maintain planar 2-D structure (Fig. 1). Polymers 1 and 2 contain six and nine nitrogen atoms per unit cell, respectively. Hence, three-electron-oxidation per unit cell of 1 leads to complete generation of semiquinone radical cation (hereafter we denote this cationic state as $\mathbf{1}[3+])$. Likely, six-electron-oxidation per unit cell of $\mathbf{2}$ leads to complete generation of semiquinone radical cation $\left(2\left[6^{+}\right]\right)$. These charged polymers are of great importance for realization of the planar 2-D high-spin organic polymers. In particular, the realization of the high-spin organic polymers with the 
layered structures such as $\mathbf{1}$ and $\mathbf{2}$ opens the possibility of the control of their spin states by facilitating the reversible and partial inclusion and/or exclusion of the counter ions like the graphite intercalation compounds. Hence, it is worth while examining the spin electronic structures of these oxidized polymers prior to the future synthetic chemical studies.

In this paper, the newly designed 2-D magnetic polymer composed of 1,3,5benzenetriamines as FC units and of para-phenylenediamines as SC units are examined on the basis of the ab initio 2-D unrestricted Hartree-Fock crystal orbital (UHF-CO) method. The aim of this study is to predict the spin electronic structures of the oxidized 2-D polymers $\mathbf{1}[3+], \mathbf{1}[6+], 2[3+]$ and $2[6+]$. 


\section{Method of Calculation}

All the calculations of the novel 2-D polymers were carried out on the basis of $a b$ initio UHF-CO method implemented in CRYSTAL98 package [18]. The 6-21G basis sets were utilized in this study [19]. To check the basis set dependency, we have performed UHF-CO calculations employing 3-21G, 4-31G and 6-31G* basis sets [19]. As a result, tendency of energy band structures remains unchanged except for the total energies.

The 2-D polymers, $\mathbf{1}$ and $\mathbf{2}$, are classified by the layer groups belonging to a subset of the 230 space groups: $P \overline{3} 1 \mathrm{~m}$ for $\mathbf{1}$ and $P 312$ for $\mathbf{2}$. The unit cells for $\mathbf{1}$ and $\mathbf{2}$ correspond to the enclosed areas in Fig. 1a and b. Prior to the UHF-CO calculations, we determined the unit cell geometry by the geometry optimizations of the model clusters (Fig. 2) on the basis of the semi-empirical molecular orbital method (AM1 [20]). Several X-ray crystal structures of the arylamine molecules relating to the present molecular structure in the unit cell have been already reported [14,21-23]. The essential features from these X-ray studies are as follows: (i) all the nitrogen atoms lie in a plane, and (ii) the 1,3,5-benzenetriyl rings are in the plane defined by the nitrogen atoms, while the para-phenylene rings are significantly canted out of the plane. The cant angle depends on the molecules studied and ranges from $40^{\circ}$ to $80^{\circ}$. However, the cant angles from the reported tetraazacyclophane molecules are within $40^{\circ}$ to $60^{\circ}$, $[14,22]$ which are in good accordance with the values $\left(59.0^{\circ}\right.$ for $\mathbf{1}$ and $47.2^{\circ}$ for 2$)$ of the AM1-geometries. In the geometry optimization process, no restriction on the cant angle of para-phenylene rings was imposed. However, 1,3,5-benzenetriyl rings were assumed to retain coplanarity with the plane defined by the nitrogen atoms. As a result, the global planarity of the present polymers can be guaranteed by the fact that all the nitrogen atoms lie in a plane, whereas the local planarity is not guaranteed owing to 
canting of para-phenylene rings. Furthermore, the other geometrical parameters were in reasonable agreement with those of the reported X-ray structures.

Finally we performed the ab initio UHF-CO calculations for the neutral and charged states of the polymers $\mathbf{1}$ and $\mathbf{2}$ employing the above-mentioned optimized unit cells. The charged unit cell for the charged polymers must be compensated by the counter ions. In the present calculations, a uniform background charge was imposed to neutralize the charge in the unit cell [18]. Note that the same unit cell geometries were used for both the neutral and cationic states. This is because the 2-D networked structures are too rigid to be easily deformed. Actually, the DFT calculations on the tetraazacyclophane molecule demonstrated that the backbone structure in the neutral state remains unchanged even in the higher charged state [14]. Therefore, the present calculations can give qualitatively reliable results for the spin electronic structures of the polymers $\mathbf{1}$ and $\mathbf{2}$. 


\section{Results and Discussion}

The geometries for the unit cells studied here are shown in Fig. 2. The bond lengths and angles take reasonable values in comparison with the reported X-ray structures of the related arylamine molecules [14,21-23]. The cant angles of the para-phenylene units in 1 and 2 are calculated to be $59.0^{\circ}$ and $47.2^{\circ}$, respectively, and therefore, the $\pi$ conjugation between the 1,3,5-benzenetriyl and para-phenylene rings is expected to become weak compared to the hypothetical planar structures of $\mathbf{1}$ and $\mathbf{2}$ (Fig. 1).

The calculated total energies per unit cell are listed in Table 1. Apparently, the high-spin states are energetically more stable than the low-spin states for the cationic forms of 1 and 2 generated by three-electron and six-electron oxidations per unit cell. Herein it should be noted that, in the $2[6+]$, all the nitrogen atoms are oxidized. In this case, it is natural that the quinoid deformation takes place and leads to diamagnetic polymers. However, this kind of deformation in the 2-D polymers is anticipated to need considerable energy compared to that in the oligomers. Therefore when the polymer 2 can be fully oxidized, $2[6+]$ is highly expected to become a high-spin polymer.

The high-spin preference for the cationic states of $\mathbf{1}$ and $\mathbf{2}$ can be explained by the band structures (Fig. 3). In $\mathbf{1}[3+]$, the $\alpha$-spin highest occupied crystal orbital (HOCO) bands (178 to 180) are less than $0.25 \mathrm{eV}$ in width (Fig. 3a). These flat bands give the physical origin for the high-spin preference. On the other hand, the corresponding frontier $\beta$-spin bands are broader than the $\alpha$-spin bands. The broadness of the $\beta$-spin bands stems from two reasons. First, the all-para-phenylene linkage exists in the polymer backbone, as is apparent from the unit cell structure in Fig. 1a. Second, 1[3+] is of partially oxidized form. Turning now to the fully oxidized $1[6+]$, the $\alpha$-spin HOCO bands (175 to 180) are less than $0.06 \mathrm{eV}$ in width, and moreover, the 
corresponding $\beta$-spin bands are also flat, originating from the fully oxidized form (Fig. 3c). The bands become so flat that the magnetic spins will be localized considerably. Again, these narrow bands give the physical origin for the high-spin preference for $\mathbf{1}[6+]$. More noteworthy is that the partially oxidized $2[3+]$ has completely flat band structures, as shown in Fig. 3 b. This contrast between $\mathbf{1}[3+]$ and $2[3+]$ is explainable by inspection of the $\mathrm{CO}$ patterns. As shown in Fig. 4a, the $\alpha$-spin HOCOs (178 to 180) for $\mathbf{1}^{[+]}$at the $\Gamma$ point are delocalized over the entire polymer backbone, whereas the $\alpha$-spin HOCOs (274 to 276) for $2[3+]$ at the $\Gamma$ point are mainly localized on the triphenylbenzene rings. Consequently, the highly localized HOCOs result in the flat bands even in $2[3+]$. In addition, the orbital patterns of the HOCOs at the $\Gamma$ point for $1[3+]$ are formed by those of the non-bonding molecular orbitals (NBMOs) of 1,3benzenediamine and 1,3,5-benzenetriamine (Fig. 4c,d). Consequently, the nonbonding HOCOs result in the flat bands in $2[3+]$.

The $\alpha$-spin HOCOs for $\mathbf{1}[6+]$ (175 to 180$)$ and $2[6+](271$ to 276$)$ at the $\Gamma$ point are shown in Fig. 5. In $\mathbf{1}[6+]$, the six spin-containing $\alpha$-spin HOCOs are separated into non-, doubly-, and triply-degenerate COs, and these COs are quasi-degenerate (Fig. 5a). Moreover, all the HOCOs in $\mathbf{1}\left[6^{+}\right]$are localized more than those in $\mathbf{1}[3+]$. On the one hand, the upper three non- and doubly-degenerate COs (274 to 276) in 2[6+] lie about 2 $\mathrm{eV}$ above the lower three non- and doubly-degenerate COs (271 to 273) (Fig. 5b). Similar to the HOCOs in $2[3+]$, those in $2\left[6^{+}\right]$have also highly localized character. However, the AO coefficients are mainly distributed to the hexaphenylbenzenetriamine moieties. As a whole, it was clarified that the nature of the HO $\alpha$-bands of the cationic forms of $\mathbf{1}$ and $\mathbf{2}$ are clearly constructed from the NBMOs of 1,3-benzenediamine and 1,3,5-benzenetriamine. 
Let us now look at the spin density distributions for $\mathbf{1}[3+], \mathbf{1}\left[6^{+}\right], 2[3+]$, and $2[6+]$, as shown in Fig. 6. In $\mathbf{1}[3+]$ and $\mathbf{1}[6+]$, most of the spin densities concentrate on 1,3,5benzenetriamine moieties due to the large cant angle of the para-phenylene rings. The spin density on the nitrogen atoms increases with going from $1[3+]$ to $1[6+]$. On the other hand, in $2\left[6^{+}\right]$, a considerable amount of spin flows into the para-phenylene rings through spin polarization effect [24], by which the positive and negative spin densities appear alternately in the two polymers. However, the spin densities on the nitrogen atoms of 1,3-benzenediamine or 1,3,5-benzenetriamine moieties are so small that we can conclude that $2[3+]$ and $2[6+]$ are spin localized systems.

The density of states (DOS) for $1[3+], \mathbf{1}[6+], 2[3+]$, and $2\left[6^{+}\right]$are shown in Fig. 7. From the calculated results, these high-spin polymers are expected to be insulating, because of a considerably large band gaps between the HOCO bands and the lowest unoccupied crystal orbital (LUCO) bands. 


\section{Conclusion}

We have investigated the electronic structures of newly designed 2-D high-spin polymers $\mathbf{1}$ and $\mathbf{2}$ composed of the strong FC unit (1,3,5-benzenetriyl and 1,3benzenediyl) and the stable SC unit (semiquinone radical cation of paraphenylenediamine). According to the ab initio UHF-CO calculations of cationic states of $\mathbf{1}$ and $\mathbf{2}$, it was found that the high-spin preference is maintained in $1[3+], 1\left[6^{+}\right]$, $2[3+]$, and $2\left[6^{+}\right]$. This indicates that the oligomer model study of $\mathbf{1}$ and $\mathbf{2}$ will be fruitful.

\section{Acknowledgements}

This work was supported by a Grant-in-Aid for Scientific Research (B) (20350065) from Japan Society for the Promotion of Science (JSPS). Numerical calculations were partly carried out at the Supercomputer Laboratory of the Institute for Chemical Research of Kyoto University. 


\section{References}

[1] N. Mataga, Theor. Chim. Acta 10 (1968) 372.

[2] A. Rajca, Chem. Rev. 94 (1994) 871.

[3] D. A. Dougherty, Acc. Chem. Res. 24 (1991) 88.

[4] W. T. Borden, E. R. Davidson, J. Am. Chem. Soc. 99 (1977) 4587.

[5] J. A. Crayton, J. N. Devine, J. C. Walton, Tetrahedron 56 (2000) 7829.

[6] A. Rajca, Chem. Eur. J. 8 (2002) 4834.

[7] Organic $\pi$-conjugated polymers having very large magnetic moment and magnetic order at low temperature have been reported: A. Rajca, J. Wongsriratanakul, S. Rajca, Science 294 (2001) 1503.

[8] T. Hughbanks, J. Am. Chem. Soc. 107 (1985) 6851.

[9] T. Hughbanks, M. Kertesz, Mol.Cryst. Liq. Cryst. 176 (1989) 115.

[10] M. Mitani, D. Yamaki, Y. Takano, Y. Kitagawa, Y. Yoshioka, K. Yamaguchi, J. Chem. Phys. 113 (2000) 10486.

[11] N. D. Mermin, H. Wagner, Phys. Rev. Lett. 17 (1966) 1133.

[12] A. Ito, A. Taniguchi, T. Yamabe, K. Tanaka, Org. Lett. 1 (1999) 741.

[13] A. Ito, H. Ino, Y. Matsui, K. Tanaka, K. Kanemoto, T. Kato, J. Phys. Chem. A $108(2004) 5715$.

[14] A. Ito, Y. Ono, K. Tanaka, Angew. Chem. Int. Ed. 39 (2000) 1072.

[15] Y. Hirao, H. Ino, A. Ito, K. Tanaka, T. Kato, J. Phys. Chem. A 110 (2006) 4866.

[16] Y. Hirao, H. Ishizaki, A. Ito, T. Kato, K. Tanaka, Eur. J. Org. Chem. (2007) 186.

[17] A. Ito, S. Inoue, Y. Hirao, K. Furukawa, T. Kato, K. Tanaka, Chem. Commun. (2008) 3242.

[18] V. R. Saunders, R. Dovesi, C. Roetti, M. Causà, N. M. Harrison, R. Orlando, C. M. Zicovich-Wilson, CRYSTAL98 User's Manual, University of Turin and Daresbury Laboratory, 1999.

[19] W. J. Hehre, P. Radom, P. v. R. Schleyer, J. A. Pople, Ab Initio Molecular Orbital Theory, Wiley, New York, 1986.

[20] M. J. S. Dewar, E. G. Zoebisch, E. F. Healy, J. J. P. Stewart, J. Am. Chem. Soc. 
107 (1985) 3902.

[21] K. Yoshizawa, A. Chano, A. Ito, K. Tanaka, T. Yamabe, H. Fujita, J. Yamauchi, M. Shiro, J. Am. Chem. Soc. 114 (1992) 5998.

[22] S. I. Hauck, K. V. Lakshmi, J. F. Hartwig, Org. Lett. 1 (1999) 2057.

[23] X. Z. Yan, J. Pawles, T. Goodson, III, J. F. Hartwig, J. Am. Chem. Soc. 127 (2005) 9105.

[24] K. Yamaguchi, Y. Toyoda, T. Fueno, Synth. Met. 19 (1987) 81. 


\section{Table 1}

Total energies (in hartree) per unit cell of the high-spin and low-spin states in the cationic forms of 2-D polymers $\mathbf{1}$ and $\mathbf{2}$.

\begin{tabular}{lrrrr}
\hline & charge per unit cell & high-spin state & low-spin state & $\Delta E_{\mathrm{L}-\mathrm{H}}{ }^{\mathrm{a}}$ \\
& neutral & -2135.280 & - & - \\
& +3 & -2135.132 & -2135.020 & 3.1 \\
& +6 & -2135.370 & -2134.936 & 11.8 \\
& & & & \\
\hline & & & & \\
& neutral & -3209.923 & -3209.803 & 3.7 \\
& +3 & -3209.940 & -3209.104 & 19.6 \\
\hline
\end{tabular}

${ }^{a}$ Energy differences (in eV) between the high-spin and low-spin states. The positive value means the high-spin preference. 


\section{Figure captions}

Fig. 1. Arylamine-based 2-D polymers; (a) 1 and (b) 2. The unit cells are enclosed by broken lines.

Fig. 2. AM1-optimized geometries of the unit cells for the ab initio SCF-UHF-CO calculations of the 2-D polymers (a) 1 and (b) 2 .

Fig. 3. Calculated band structures along the $\Gamma-\mathrm{M}$ direction of the Brillouin zone for the high-spin states of (a) $\mathbf{1}[3+]$, (b) $2[3+]$, (c) $\mathbf{1}\left[6^{+}\right]$, and (d) $2\left[6^{+}\right]$. The broken lines designate the Fermi level.

Fig. 4. $a$-spin HOCO patterns at the $\Gamma$ point for the high-spin states of (a) $1[3+]$ and (b) $2[3+]$, and the schematic NBMOs of (c) 1,3-benzenediamine and (d) 1,3,5benzenetriamine. The $\mathrm{CO}$ patterns are depicted after the expansion of the actual molecular structures onto the plane of the page. The radii of the black and white circles corresponds to the coefficients of $p$-type atomic orbitals (AOs) and the only AOs with their coefficients larger than 0.02 are shown in this figure. The HOCOs can be regarded as psuedo- $\pi$-bands.

Fig. 5. $\alpha$-spin HOCO patterns at the $\Gamma$ point for the high-spin states of (a) $1[6+]$ and (b) $2[6+]$. The CO patterns are depicted after the expansion of the actual molecular structures onto the plane of the page. The radii of the black and white circles corresponds to the coefficients of $p$-type atomic orbitals (AOs) and the only AOs with their coefficients larger than 0.02 are shown in this figure. The HOCOs can be regarded as psuedo- $\pi$-bands. 
Fig. 6. Schematic representation of the Mulliken spin density distributions of (a) $\mathbf{1}[3+]$, (b) $1[6+]$, (c) $2[3+]$, and (d) $2[6+]$. They are depicted after the expansion of the actual molecular structures onto the plane of the page. The solid and white circles show the positive and negative spin densities, respectively, and their radii are in proportion to the magnitudes of the spin density. Only the spin density larger than 0.05 is shown in this figure.

Fig. 7. The density of states for the high-spin states of (a) $\mathbf{1}[3+]$, (b) $2[3+]$, (c) $1\left[6^{+}\right]$, and (d) $2[6+]$. The arrows designate the Fermi level. 
a

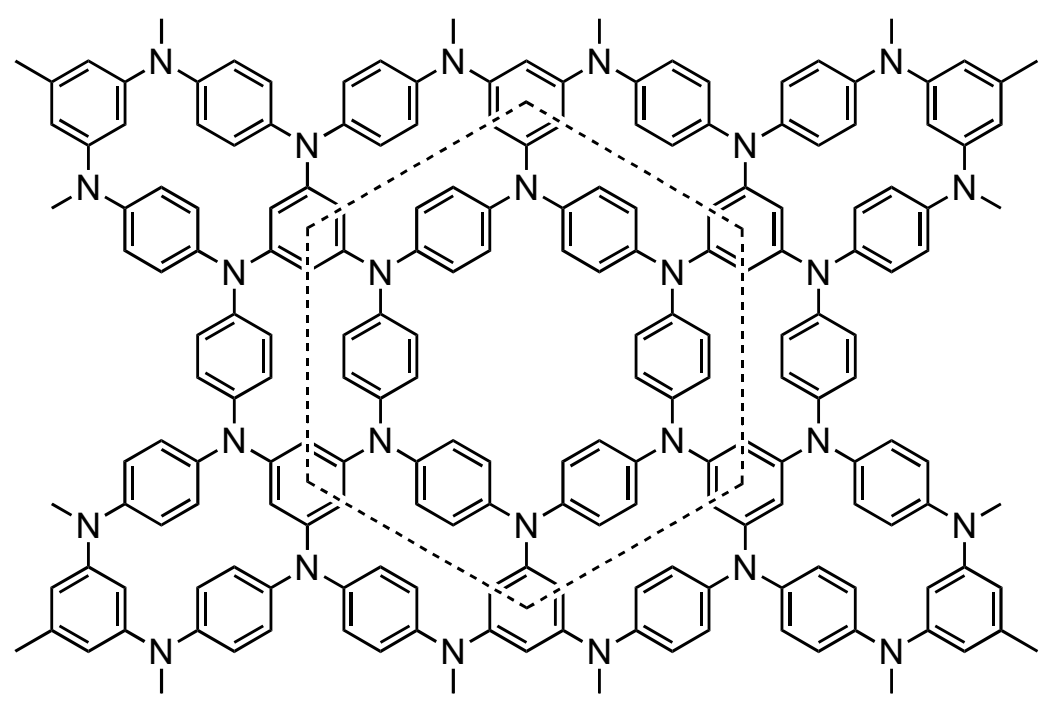

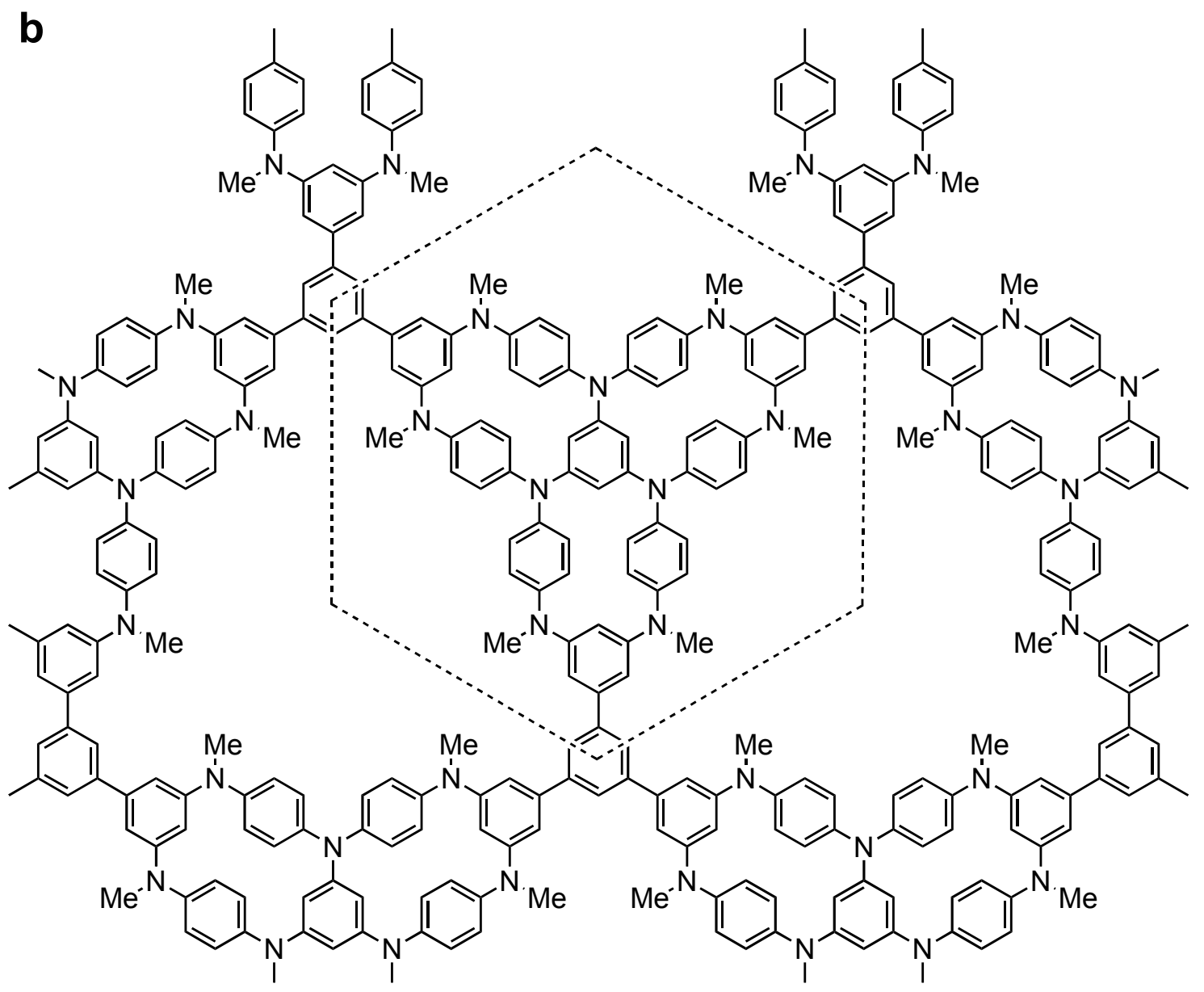

Fig. 1. 
a

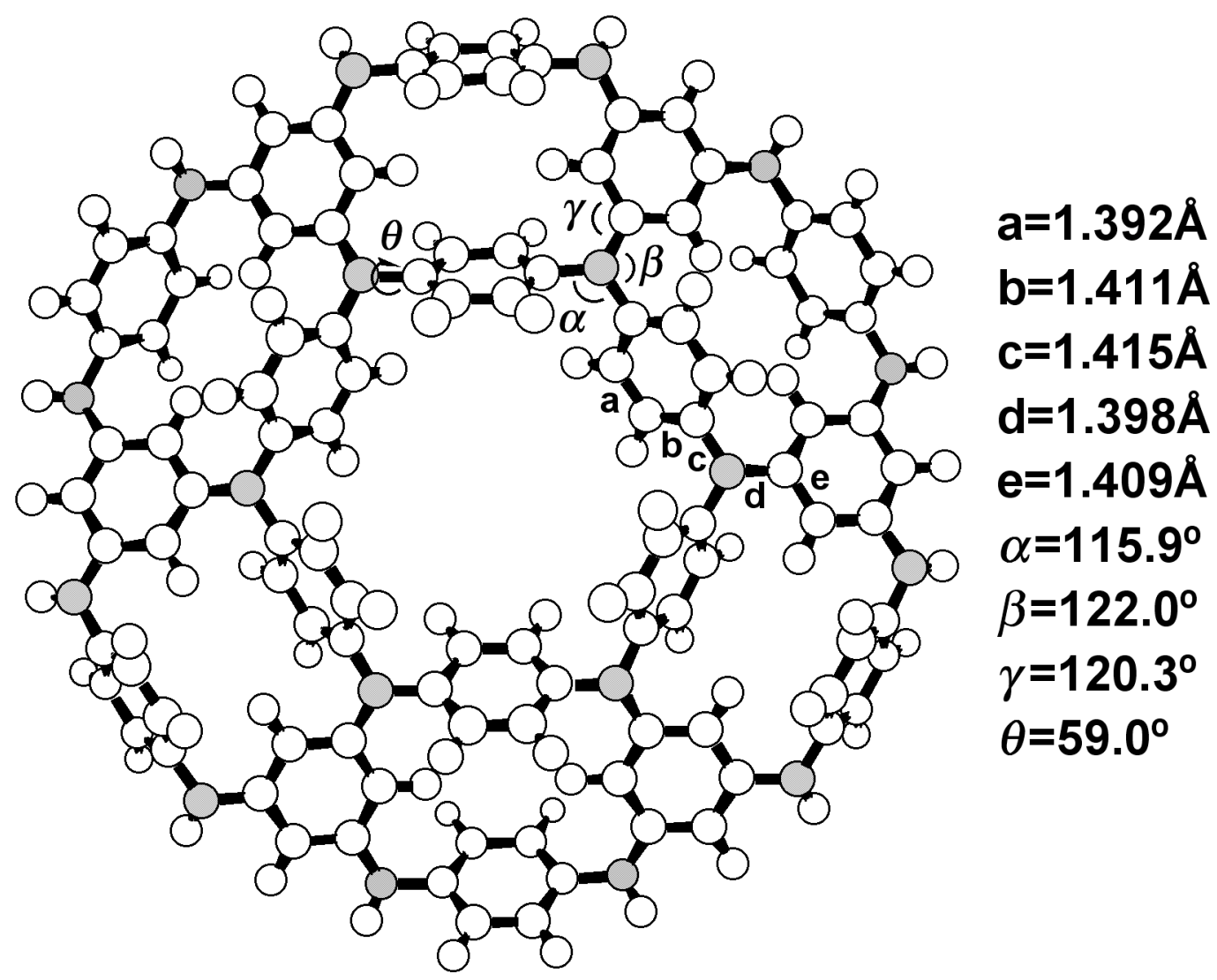

b
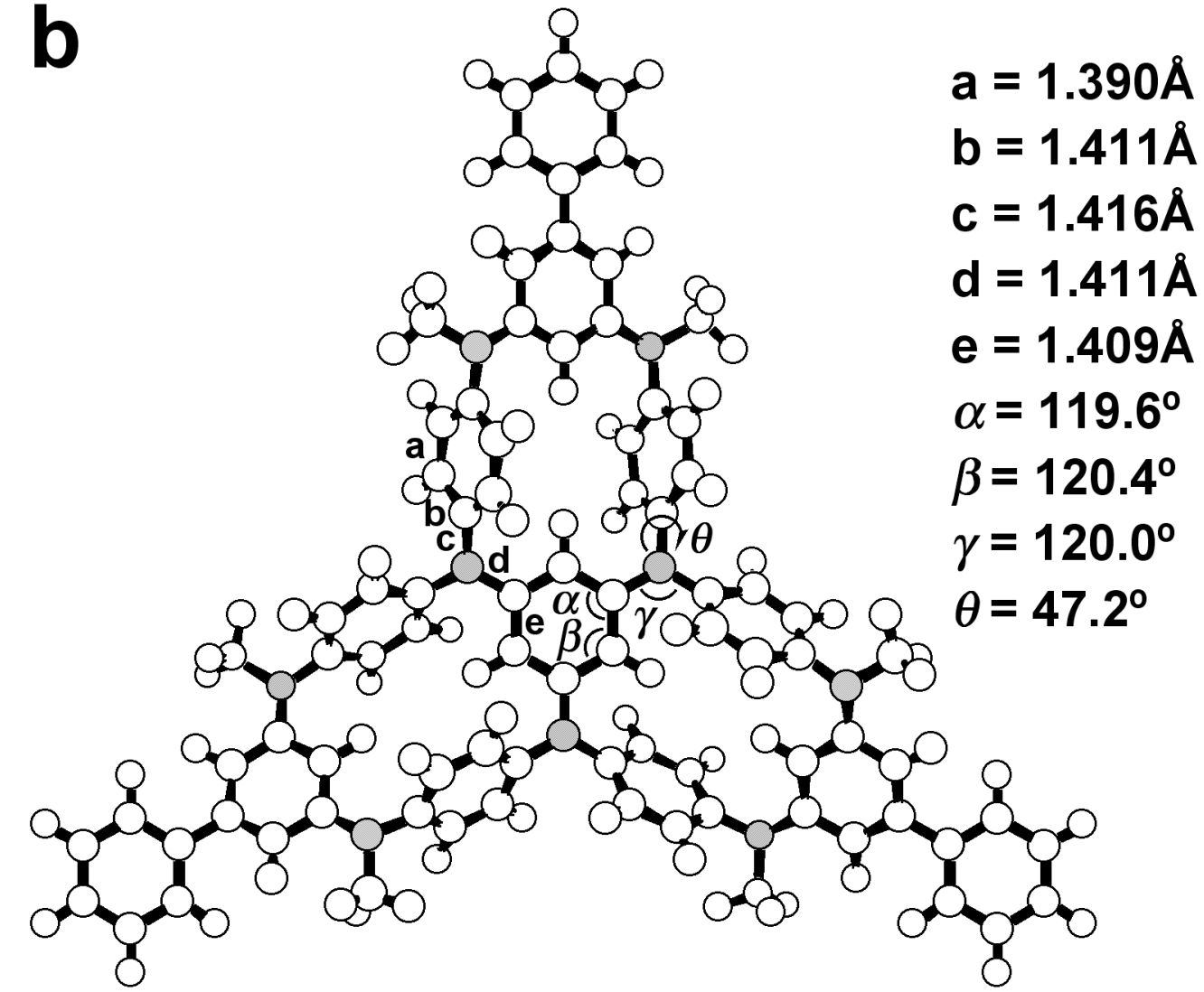

Fig. 2.

$$
\begin{array}{ll}
a=1.390 \AA \\
b=1.411 \AA \\
c=1.416 \AA \\
d=1.411 \AA \\
\end{array}
$$



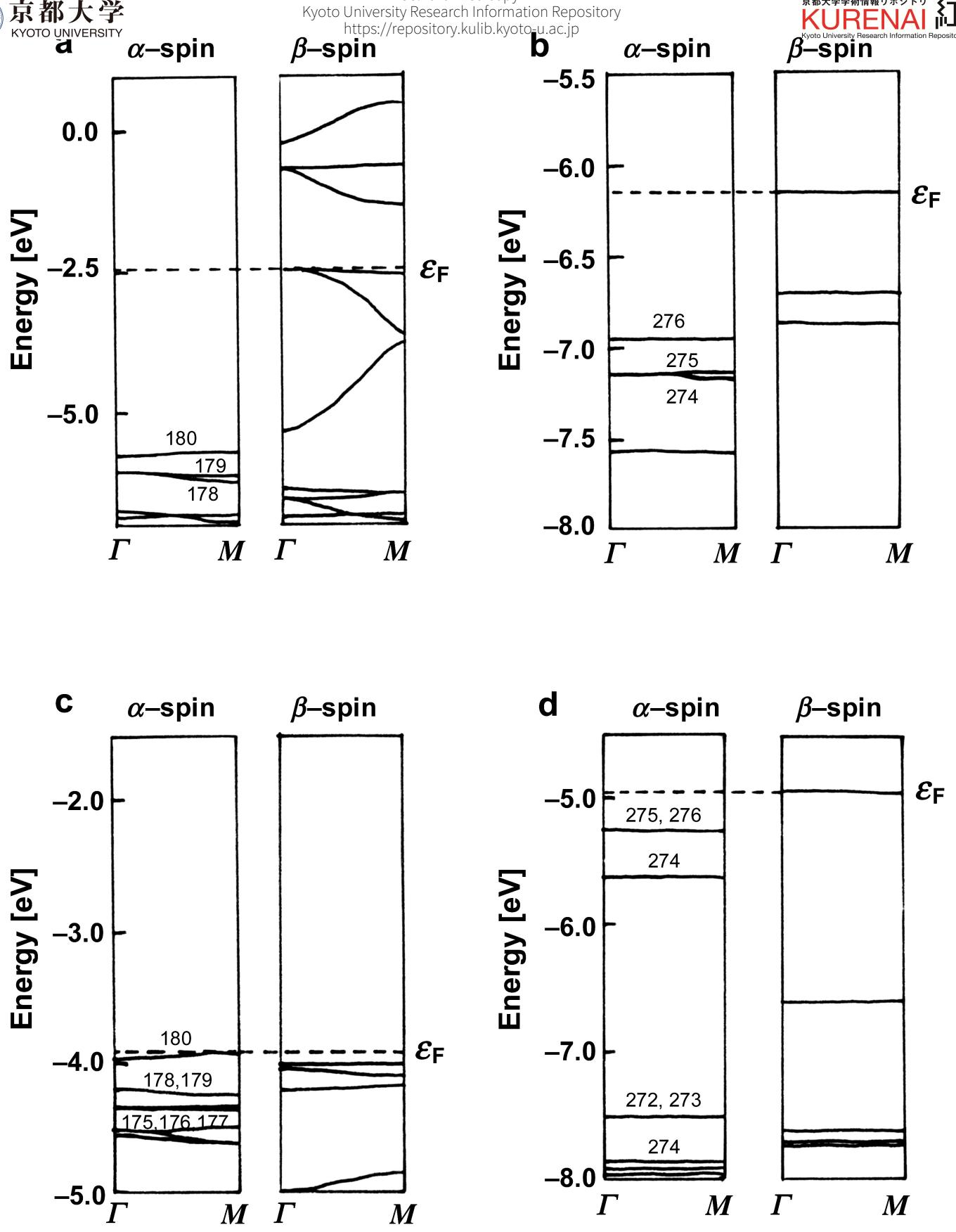

Fig. 3. 
a

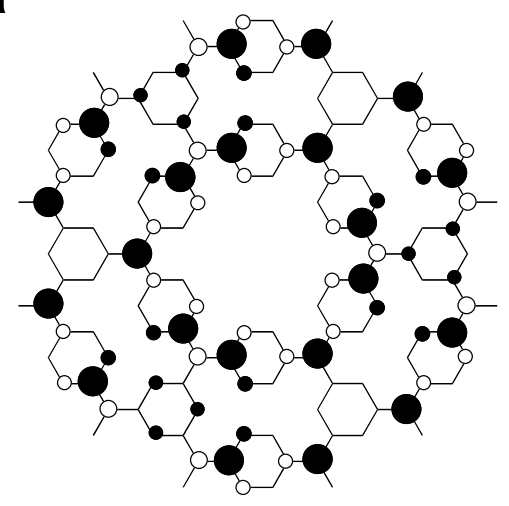

180

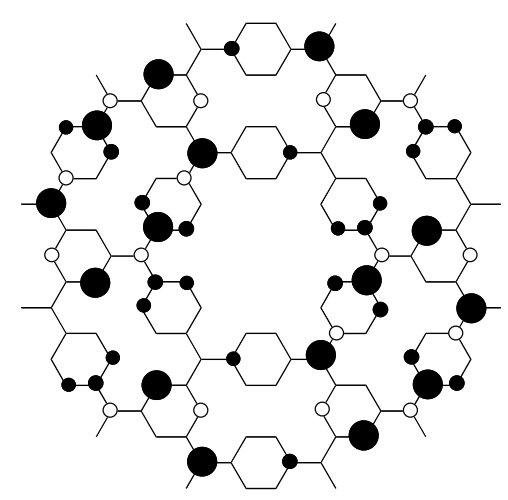

179

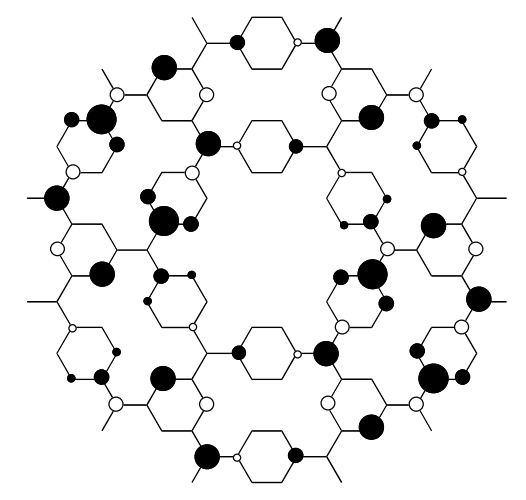

178

b

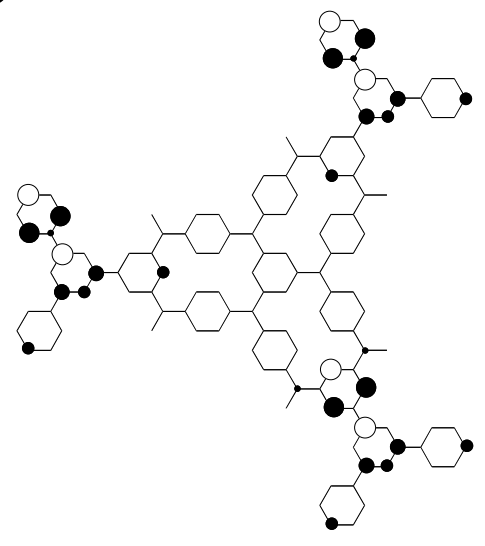

276

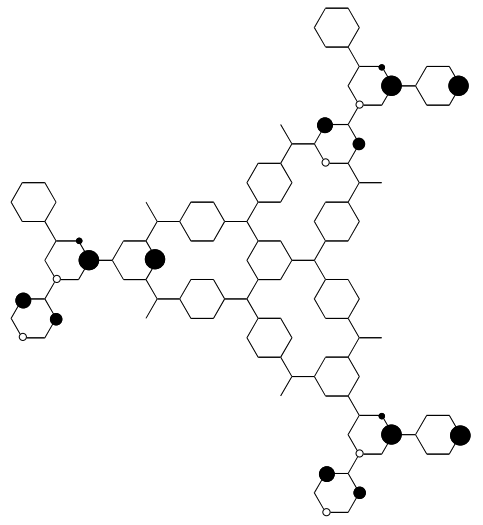

275

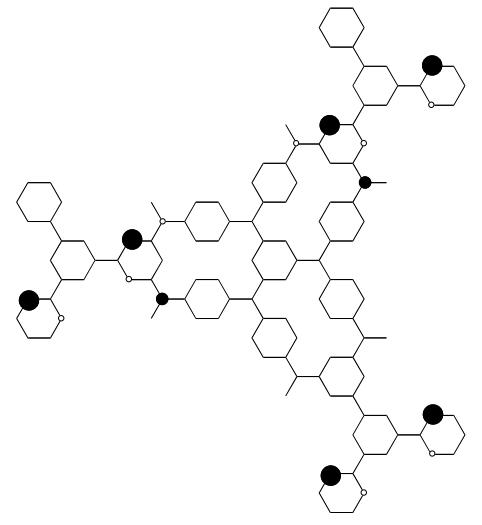

274

C<smiles>CC1CC(O)[C@H](O)CC1O</smiles>

d

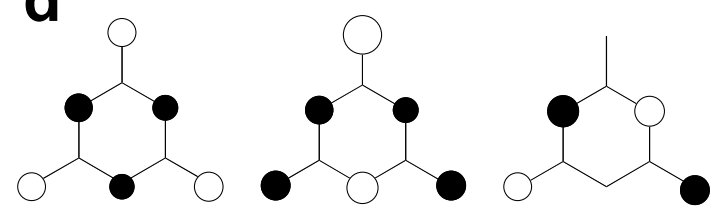

Fig. 4. 


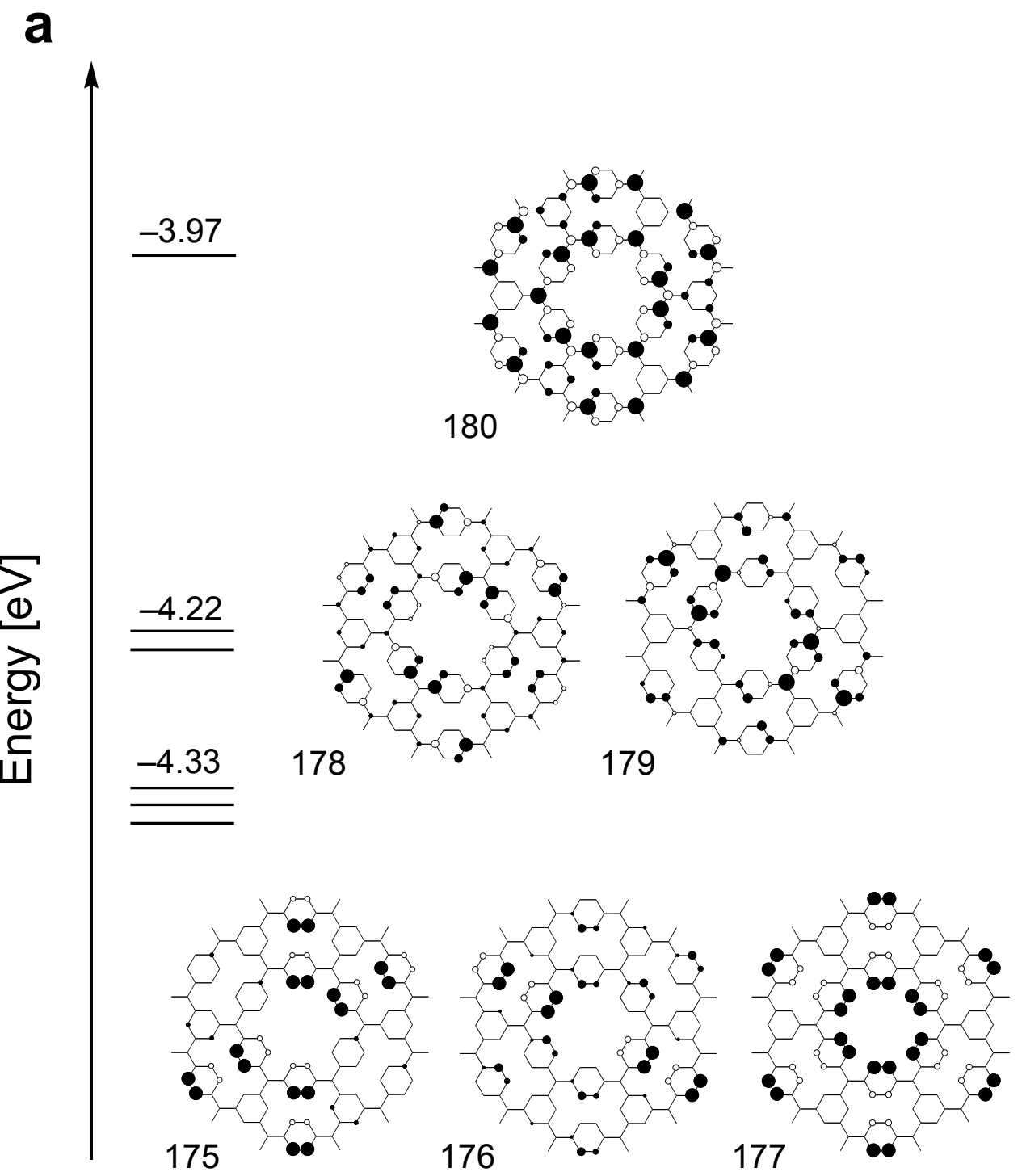

Fig. 5a 


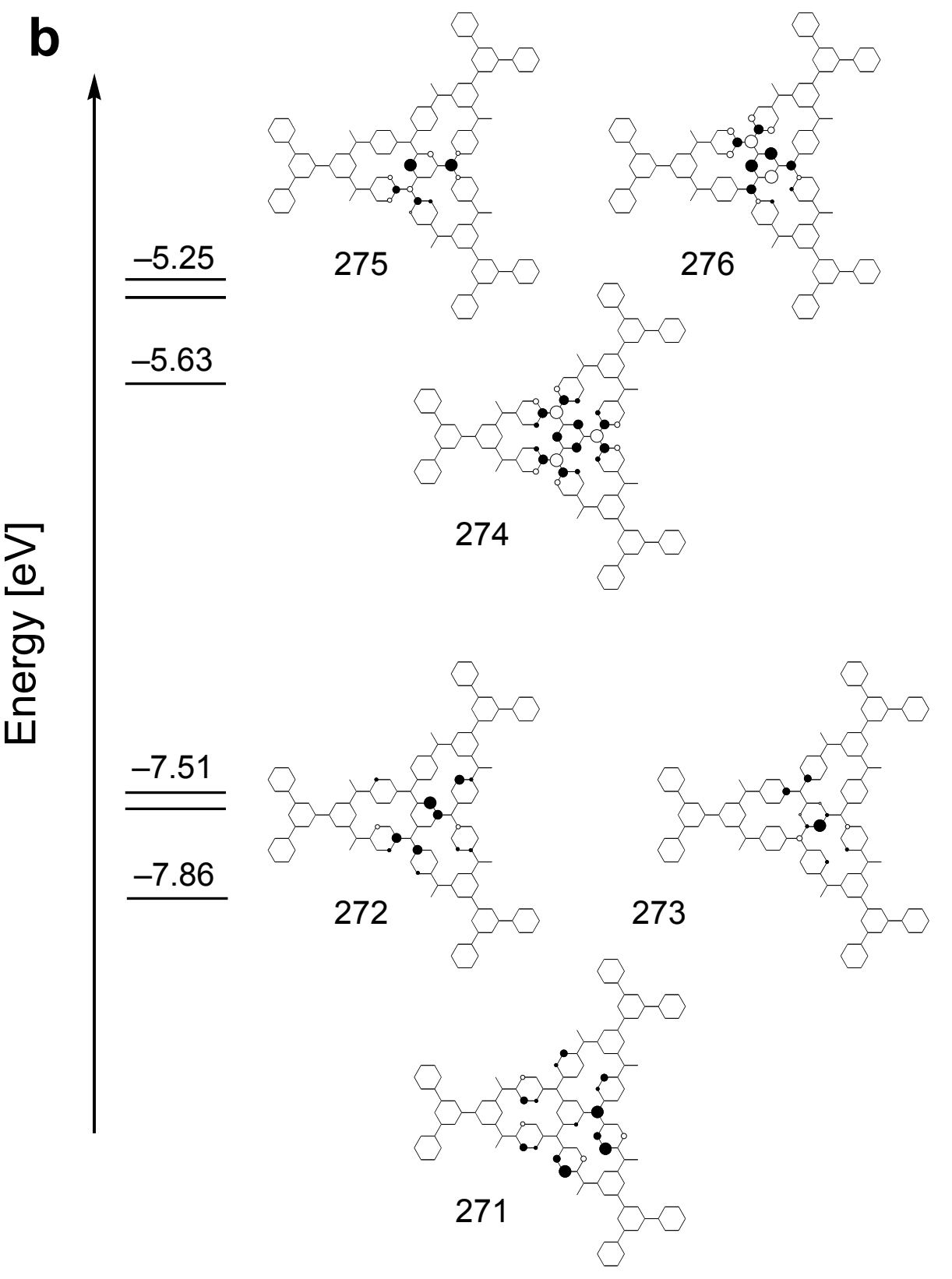

Fig. $5 b$ 
a

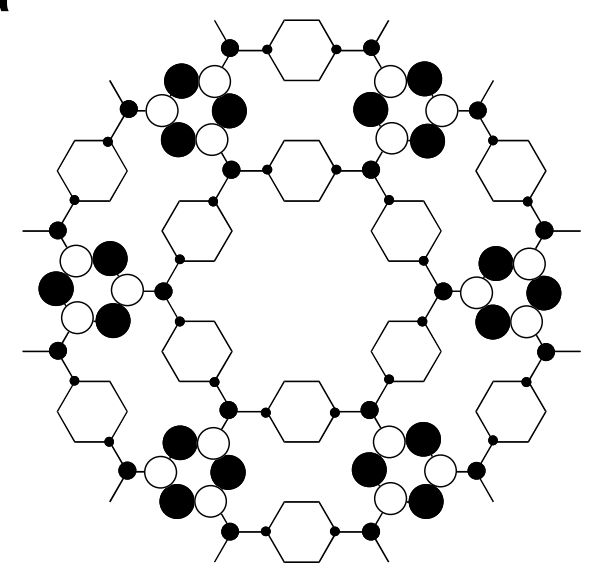

C

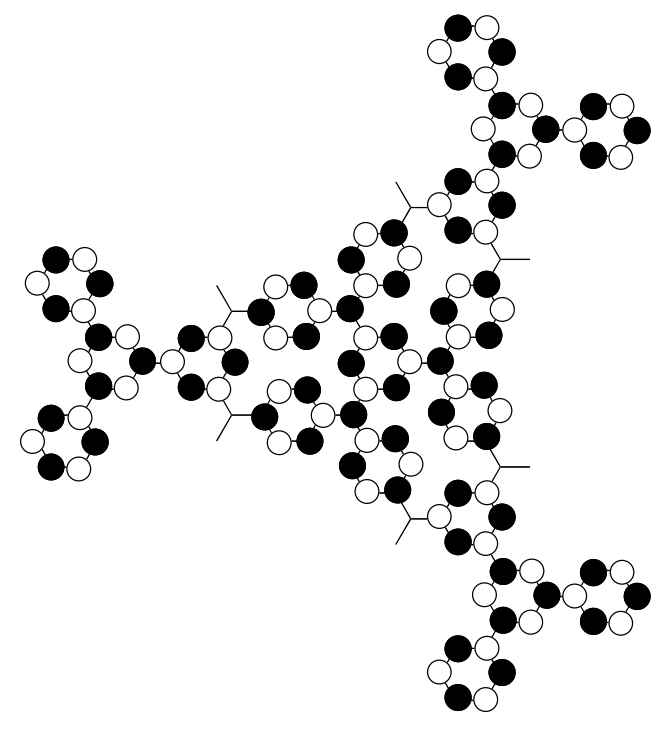

b

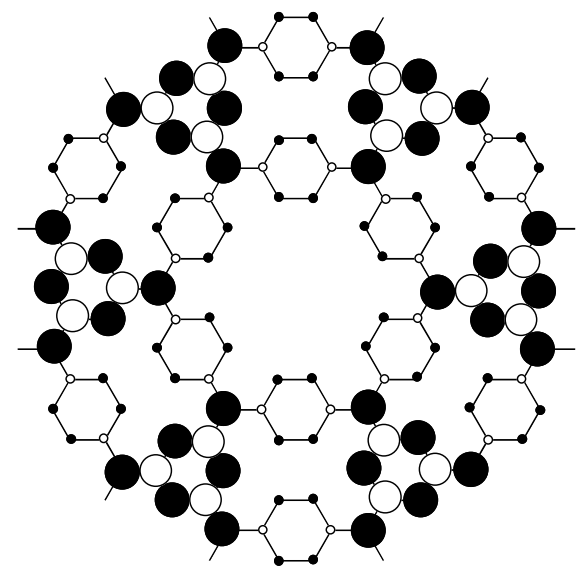

d

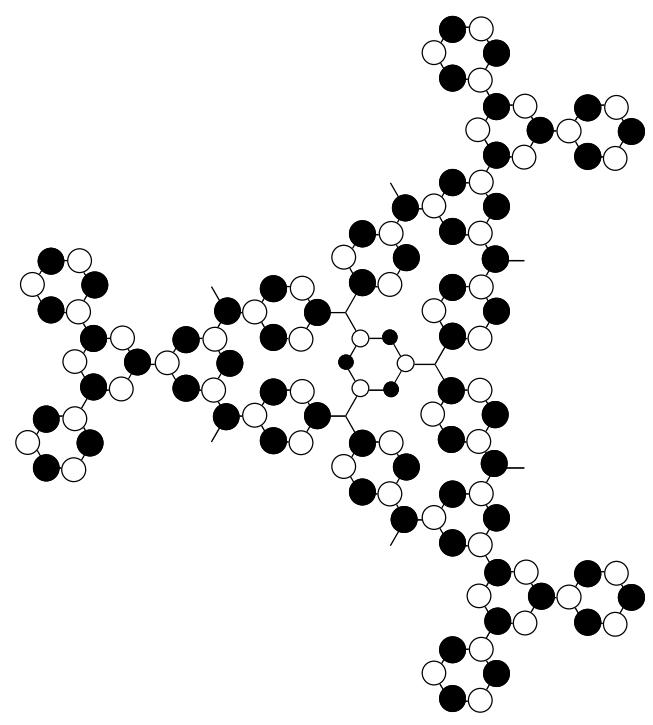

Fig. 6. 
a

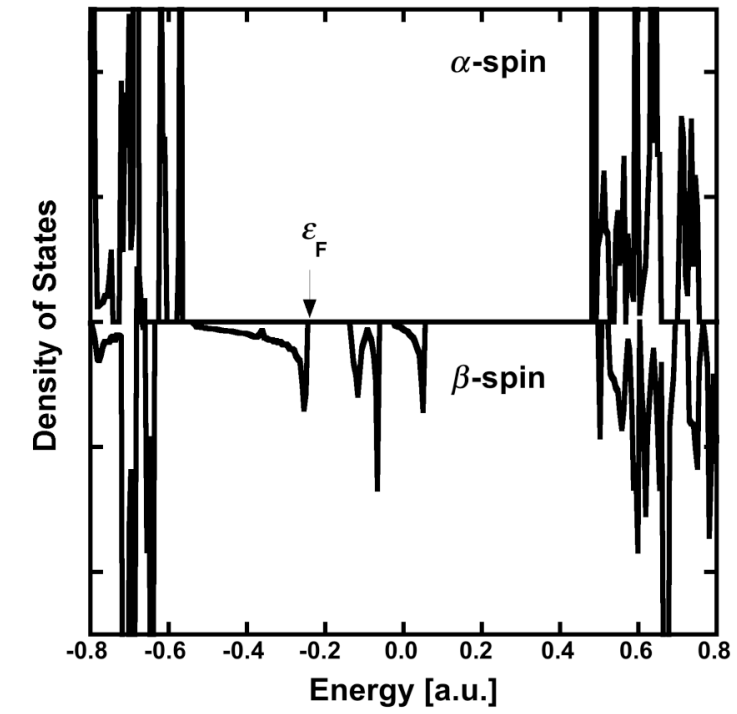

C

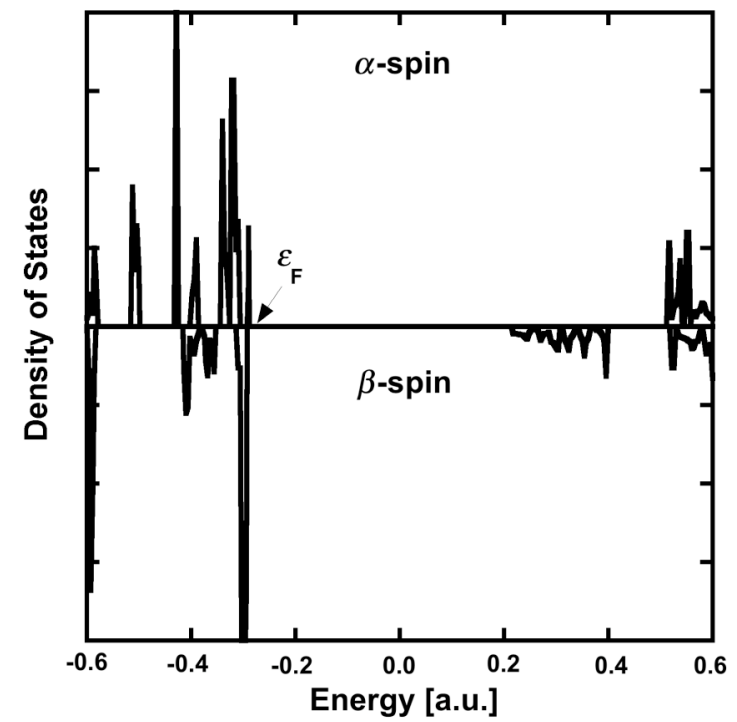

b

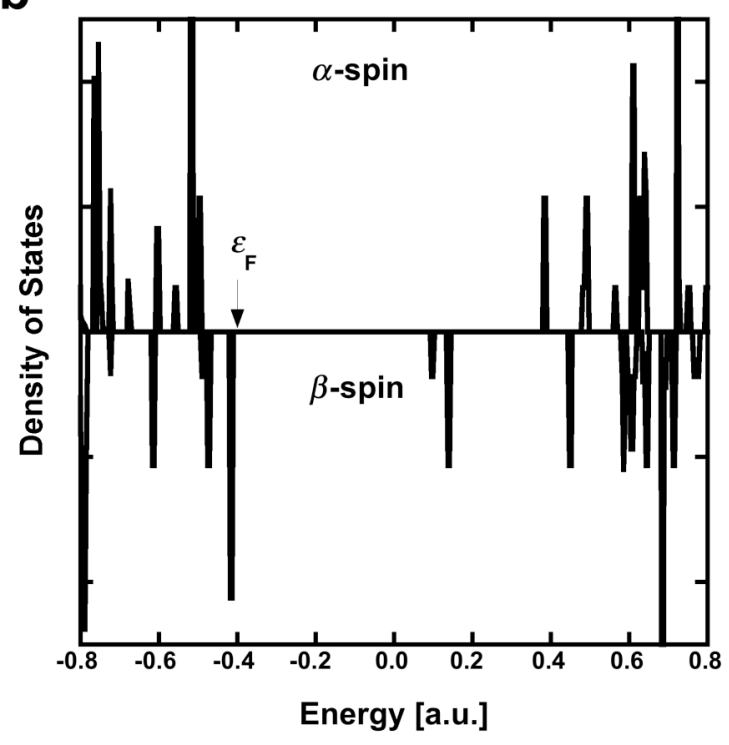

d

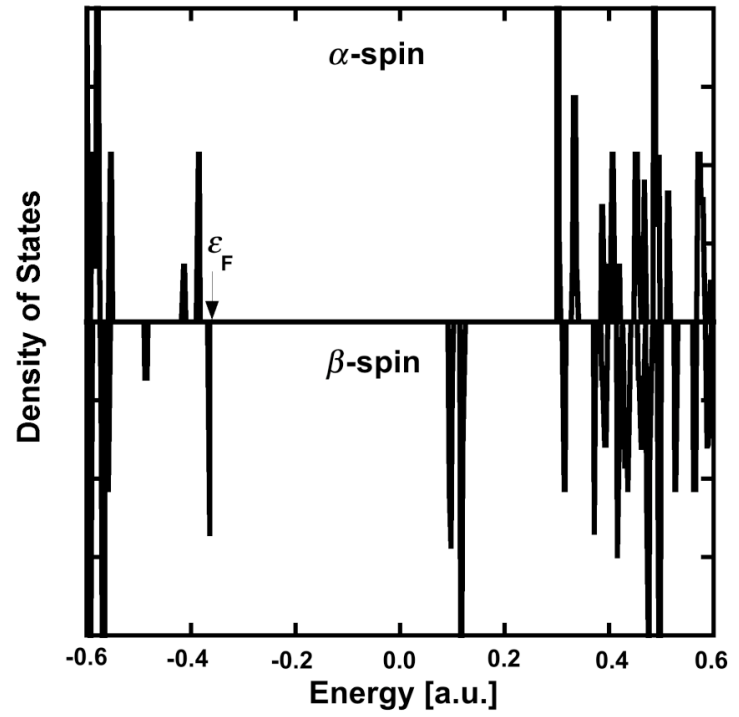

Fig. 7. 